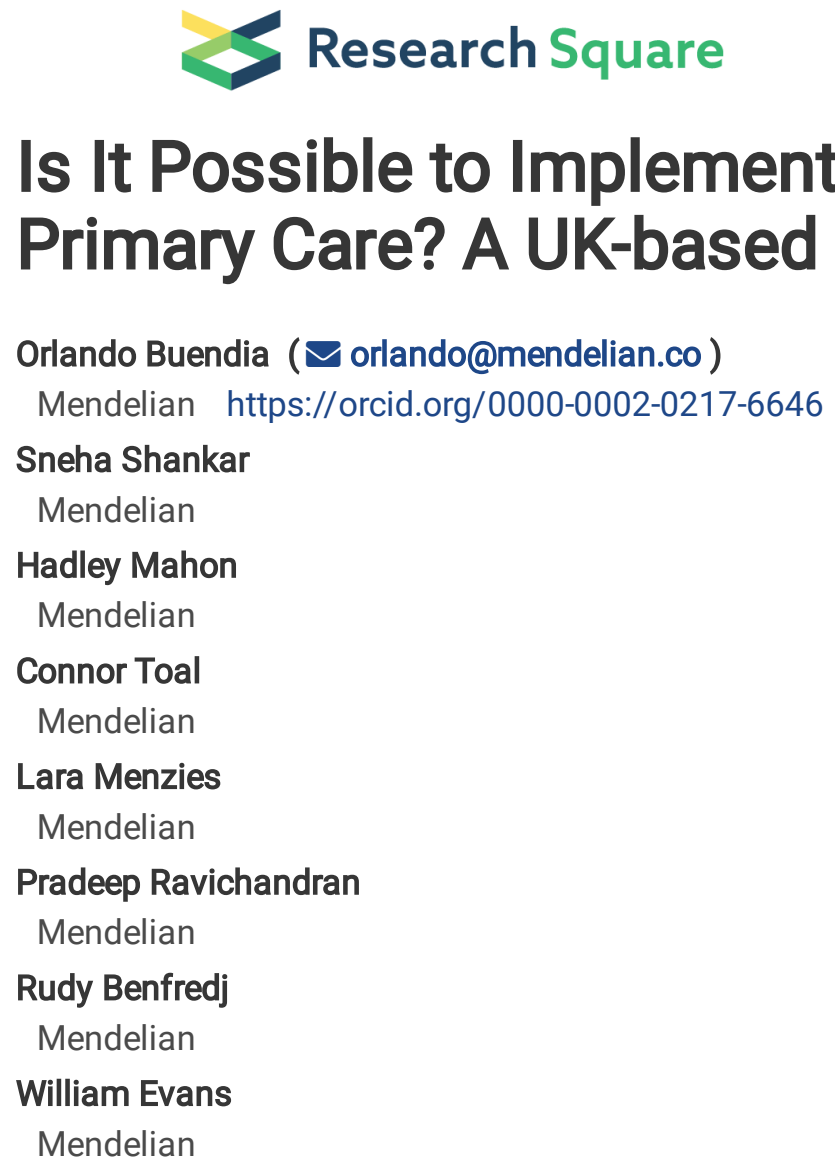

\title{
Is It Possible to Implement a Rare Disease Case-finding Tool in Primary Care? A UK-based Pilot Study
}

Research

Keywords: Rare disease, primary care, electronic health records, database analysis

Posted Date: June 30th, 2021

DOI: https://doi.org/10.21203/rs.3.rs-638180/v1

License: (a) (i) This work is licensed under a Creative Commons Attribution 4.0 International License. Read Full License 


\section{Abstract}

\section{Introduction:}

This study implemented MendelScan, a primary care rare disease case-finding tool, into a UK NHS population. Rare disease diagnosis is challenging due to disease complexity and low physician awareness. The 2021 UK Rare Diseases Framework highlights a global need for faster diagnosis to improve clinical outcomes as a key priority.

\section{Methods \& Results:}

A UK primary care locality with 68,705 patients was examined. MendelScan encodes diagnostic/screening criteria for multiple rare diseases, mapping clinical terms to appropriate SNOMED CT codes (UK primary care standardised clinical terminology) to create digital algorithms. These algorithms were applied to a pseudo-anonymised structured data extract of the electronic health records (EHR) in this locality to "flag" at-risk patients who may require further evaluation. All flagged patients then underwent internal clinical review; for those that passed this review, a report was returned to their GP.

55 of 76 disease criteria flagged at least one patient. 227 ( $0.33 \%$ of the total population) patients were flagged; 18 EHR were already diagnosed with the disease. 75/227 (33\%) passed our internal review. Thirty-six reports were returned to the GP. Feedback was available for $28 / 36$ of the reports sent. GP categorised nine reports as "Reasonable possible diagnosis" (advance for investigation), six reports as "diagnosis has already been excluded", ten reports as "patient has a clear alternative aetiology", and three reports as "Other" (patient left study locality, unable to reidentify accurately). All the 9 cases considered as "reasonable possible diagnosis" had a further actionable evaluation.

\section{Conclusions:}

This pilot demonstrates that implementing such a tool is feasible at a population level in an ethical, technical and efficient manner. The case-finding tool identified credible cases which were subsequently referred for further investigation. Future work includes performance-based validation studies of diagnostic algorithms and the scalability of the tool.

\section{Background}

Rare diseases (RD) are individually rare but collectively common [1], with an estimated of 6,000-8000 RD and affecting 3.5$5.9 \%$ or $263-446$ million persons globally [2]. RD are heterogeneous in aetiology, frequently chronic and debilitating [3]. There is no universal definition of a rare disease, with most legislative frameworks using point prevalence. In the UK and the European Union (EU) a rare disorder is defined as affecting fewer than 1 in 2000 persons [4,5]. 71.9\% are considered genetic diseases and $69.9 \%$ have a pediatric-onset [2]. Of all RD, 149 diseases (4.2\%) have a prevalence in the range of 1-5 per 10,000, these account for $77.3-80.7 \%$ of the total population of patients affected. Collectively RD are a significant burden to healthcare systems and society, the economic burden of 379 RD with a combined prevalence of 15.5 million in the US, was estimated to be $\$ 966$ billion in 2019. [6].

Rare disease diagnosis is challenging meaning that patients frequently remain without a correct diagnosis for extended periods. This hunt for a diagnosis has its own term: the diagnostic odyssey [7]. During this diagnostic odyssey, patients typically experience numerous primary care visits, specialist clinic reviews, investigations, invasive interventions, misdiagnoses and inappropriate treatments [8]. A cohort of patients with RD in the UK and US reported a diagnostic delay of an average 5.6 and 7.6 years respectively, with patients typically visiting eight physicians (four primary care and four specialists) and receiving two to three misdiagnoses [9]. Similarly an EU survey reported that $40 \%$ of patients with RD were initially incorrectly diagnosed, and a quarter experienced a diagnostic odyssey of more than 5 years [10].

The reason for this diagnostic delay is multifactorial with issues related to the heterogeneity of many rare diseases, clinicians and healthcare systems. No individual clinician can be expected to know all RD, and the adage "When you hear hoofbeats, do not expect to see Zebras" [11], describes a well held approach to considering the differential diagnosis of a clinical problem, but 
is not helpful for diagnosing patients with RD. Enabling clinicians, especially those in primary care, to identify unusual patterns and revisit diagnoses is crucial to reducing the diagnostic odyssey for patients with RD [12].

Early diagnosis is central to achieving better patient outcomes [13]. It enables an improved assessment of prognosis, optimization of care, access to therapies, linkage to patient organisations, easier access to social and educational support, as well as more accurate disease information [12]. It also brings clarity and understanding to the challenging, puzzling and costly diagnostic odyssey for patients and their families [14]. Furthermore, an accurate diagnosis enables the patient to contribute to the broader understanding of their disease, through for example patient registries, engagement with research and therapy development [15].

In the UK, addressing this diagnostic odyssey is the first of four key priorities in the The UK Rare Diseases Framework, published in January 2021. The priority 'Helping rare disease patients get a final diagnosis faster' is underpinned by five themes and proposes using data and digital technologies as a solution to enable a more timely diagnosis [16].

\section{Methods}

Mendelian is a UK-based health data analytics company focused on shortening the diagnostic odyssey of rare and hard-todiagnose diseases. Mendelian has developed a digital case-finding tool, "MendelScan", that can analyse structured clinical vocabulary, such as SNOMED CT codes [17] from primary care electronic health records (EHR) and highlight patterns of data that correspond to an increased likelihood of the patient being affected by certain RD. This enables the identification of those at risk and assists their clinician in accessing the correct diagnostic pathway. The MendelScan system is summarised in Figure 1.

The pilot study took place between January 2019 and October 2020. The primary objective was to assess the feasibility of applying MendelScan at a small scale in a primary care environment in the Lower Lea Valley (LLV) primary care GP Federation.

The process for delivering MendelScan into the selected primary care federation involved establishing agreements, deploying the algorithms into a pseudonymised data set, manually reviewing the EHR identified by the algorithm, delivering the reports to GP and collecting their feedback. Figure 2 summarises the implementation process.

\subsection{Primary care EHR access}

\subsubsection{Ethics and information governance}

To enable data access and confidence in this study an independent ethical analysis of this approach was commissioned [18]. Building on the outcome and recommendations of this report, and in compliance with information governance legislation, a data sharing agreement (DSA) was agreed between stakeholders. The DSA is a contract that stipulates the rules regarding usage and handling of data. Finally, a Data Protection Impact Assessment (DPIA) was drafted. identifying and minimising the data protection risks of the project [19].

\subsubsection{Data Transfer}

Data transfer involved creating a data set of patients' EHR, removing personal identifiers (name and address) for these records and creating a pseudonym, a unique numeric identifier; Individuals who had opted out of sharing such data, through the national data opt-out, were removed. The pseudonymised records of all other patients were extracted and sent to Mendelian for analysis.

\subsection{Algorithm deployment}

Mendelian developed an approach to build algorithms for each RD in the following steps: 
1. Analysing the suitability of a RD using a scoring system based on features of the disease, the benefit of early diagnosis and the likelihood that relevant clinical characteristics would be captured in the primary care EHR.

2. Performing a systematic literature review, searching for peer-reviewed screening or diagnostic criteria for the selected RD.

3. Digitising the selected criteria into a numeric algorithm using structured data SNOMED CT codes, based on a combined scoring across several individual data points (Table 1). We did not interrogate data held in unstructured formats such as letters or free text in clinic notes.

The algorithms were deployed in the pseudonymised EHR data extracts. The MendelScan case-finding tool checked the algorithms against the data extract and highlighted all those that were above the suspicion threshold. MendelScan included seventy-six RD. (See Appendix 1)

The extracted EHR dataset consisted of structured data (SNOMED CT) codes. Each EHR was represented by different types of information codes given in Table 1.

\subsection{Review of identified EHR}

The review process is summarised in Figure 2.

We performed an anonymous, two-round manual review process for each EHR identified by any of the seventy-six algorithms deployed. In round one, a medical doctor reviewed each EHR and assigned each case one of three outcomes: (1) rule-in, (2) ruleout (3) already diagnosed. In round two, rule-in cases were further reviewed by a GP, geneticist or an expert in a particular rare disease and further assigned a rule-in or rule-out outcome. For each rule-in case, a patient report was generated and sent to their GP practice.

\subsection{Send reports to GP}

The reports of each of the rule-in patients were returned to their GP by email. Each report included the unique patient identifier that enabled matching to the patient's full EHR at the practice.

Each report included an explanation of the condition identified, the reasons for highlighting this disease for this patient, and the next steps for further evaluation after GP review.

\subsection{GP feedback on reports}

Feedback from GP was requested at two different stages. The first, patient report feedback, was requested as soon as the GP completed evaluating each patient's report and full EHR. It consisted of an online questionnaire, accessed through a link on each patient report, with three multiple-choice questions and one open-ended question. (See Appendix 2)

The second, patient outcome feedback, was requested 3 months after the initial feedback and focused on all cases where the GP indicated that the report suggested a reasonable possible diagnosis and were advanced for further investigation in Figure 5 .

\section{Results}

The results of the implementation process are summarised in Figure \# 6.

\subsection{Primary care EHR access}




\subsubsection{Ethics and information governance}

The ethics report helped to elucidate that the project was ethical and identified potential risks in data management for each stakeholder to minimise. The information governance process (Data Sharing Agreement and the Data Protection Impact Assessment) took almost a year to complete. There were no standard documents to enable data transfer for commercial service providers in the UK NHS. With no standardised documents nor any previous experience of such processes in either Mendelian nor the LLV, this took longer than anticipated to agree the legal and information governance documentation.

\subsubsection{Data transfer}

The process of data transfer led to about one-third reduction in the number of EHR due to either no clinical events being recorded, death of the patient, deregistration or issues related to the quality of the data (incomplete or an unsound record). After the data cleaning process, the de-anonymised data set included 68,705 patients' EHR from a single primary care locality.

\subsection{Algorithm deployment}

All 76 algorithms were deployed successfully into the de-anonymised data set. 55 algorithms identified at least one EHR. 227 ( $0.33 \%$ of the total population) EHR were identified in total; $18 \mathrm{EHR}$ had an existing diagnostic code for the RD intended to identify. 152 EHR were found with a diagnostic code for any of the 76 rare diseases investigated.

\subsection{Review EHR identified}

75 of the 227 EHR (33\%) passed our internal review process.

\subsection{Send reports to GP}

36 of the 75 (48\%) patient reports were returned to the GP practices. The return of reports was batched to manage GP workload (a maximum of 5 reports per week per GP practice). A GP Federation Research Nurse, with access to the full EHR across multiple GP practices, did an initial review of reports for the majority of the practices in the study.

Feedback reported that: the batching of reports was welcomed both to manage the workload and enable the review work to be planned efficiently; the reports were re-identified within the primary care data system successfully; and found to be clear and laid out with the reasons for flagging that patient and supporting evidence for this easy to interpret, enabling a quick and targeted approach to challenge/confirm the conclusions.

\subsection{GP feedback on reports}

The first feedback was currently available for 28 of the 36 reports sent to the GP at the time of publishing.

Second feedback was currently available for six of the nine patients at the time of publishing who went on for further evaluation. The summary of the second feedback outcomes is in table 5 .

\section{Discussion}

While this pilot was limited in scope, it demonstrated the potential of this approach in three critical areas:

1. It validated that a rare disease case-finding program could be designed within appropriate guidelines for data privacy and protection.

2. It proved the feasibility of using phenotypes documented within EHR as the basis for case finding algorithms. 
3. It revealed that rare disease case finding is possible without significant disruption to the GP workflow or local specialist referral volumes.

There are two additional elements critical to the overall potential of EHR case finding that weres not demonstrated in this pilot. The first is the clinical validity of the algorithms in the form of a statistically significant positive predictive value or other measures of sensitivity and specificity. This pilot was not powered to demonstrate this. Given the incidence of rare diseases, it is unlikely any pilot could be of sufficient size to determine the validity of an individual disease algorithm. However, significant potential exists in future work to demonstrate the validity of a multi-disease portfolio of algorithms or of individual disease algorithms retrospectively in a large database. This work is ongoing.

The second element not demonstrated is the scalability of the approach. To demonstrate scalability in the future, we will need to show the ability to :

- Gain direct access to dynamic EHR in an appropriate, pseudonymised form .

- Demonstrate value to patients and efficiency for GP.

- Maintain quality with reduced reliance on manual review by internal clinicians.

\subsection{Challenges and barriers for digital health deployment in rare diseases.}

\subsubsection{Data access considerations}

The uptake of digital health solutions is hindered due to considerations related to data privacy, IT systems security, and data confidentiality. These are partly due to technical inability to hold the data safely or due to the dynamic aspect of the legal framework. Finally, the scarcity of reimbursement models for digital health services have limited the potential adoption of technical solutions and their widespread use across the NHS.

In the current environment, it remains difficult to share and transfer patient health information, among healthcare professionals, from different organisations. This prevents a seamless care environment and breaks the promise of a potential continuity of care across the spectrum of the caregivers and predisposes the system to delays in diagnosis.

\subsubsection{Primary care EHR}

To enable pseudo-anonymisation of the EHR data only structured/coded data was extracted. This structured data can be considered to be more accurate with numerical values such as weight, height, blood pressure and laboratory values readily comparable from one patient to the next. Diagnoses and clinical features are typically captured by physicians in UK primary care. Those that have been encoded and therefore appearing in a structured data extract, are typically those that are recorded with a greater degree of confidence, free text entries often recording such information if there is greater uncertainty. Consequently, one can consider the quality of this coded data as a strength, however, this means that the majority of information in the EHR was not examined by MendelScan. Free text diagnoses, clinical features and the content of secondary care correspondence, often rich in information, was largely not examined, with such correspondence often being captured in the structured data as the presence of the letter and sometimes a single diagnostic code. The motivators and context for coding in primary care also needs to be considered, including the demonstration that certain tasks have been performed for reimbursement (e.g. Quality and Outcomes Framework) and to have pertinent information quickly to hand for future consultations.

\subsubsection{Physician-related barriers for digital health deployment}

Page 6/10 
The implementation of any digital health tool into routine clinical practice faces challenges. In this study some practices who initially agreed to be involved withdrew due to being overwhelmed by routine practice workload to entertain a new technology such as MendelScan. For successful adoption after passing any initial resistance to engagement, design and functionality are key, with systems poorly designed or implemented shown to create cognitive overload and disrupt workflow [20,21]. To this end, a key implementation decision for this study was to not directly integrate the returning of flagged reports into the EHR as an alert or "pop-up". Alert fatigue, the diminishing return on the utility of alerts with their increased use, was a concern in a system such as MendelScan that would add to already existing alerts within the EHR [22]. Decision making about identified cases was not immediately time critical, so we chose to return reports in a format that could be actioned at a time convenient to the clinician.

The COVID-19 pandemic significantly affected the implementation of this pilot, protracting its roll-out, contracting and practices dropping out due to additional clinical pressures. Further, the restructuring of primary care organisations during the pilot led to further disruption with contracting and data sharing agreements and responsibilities needing to change. Health care services are not static organisations and such changes need to be considered. Despite this, some of the restructuring changes such as the introductions of integrated care systems and organisations being challenged to improve public health across larger populations of 1-2 million, may be well placed for encouraging such rare disease case finding. Also, the COVID-19 pandemic has led to the wider adoption of digital health technologies and the blending of these into routine healthcare pathways in the NHS; we are optimistic that this will help facilitate the use of technologies such as MendelScan in the future.

\section{Conclusions}

Overall, this pilot demonstrates that implementing a novel digital case-finding tool for RD in primary care in the UK is feasible in an ethical and workable manner at a population level. The pilot also demonstrates a minimal impact on workload or system resources which is acceptable to users. The digital case-finding tool identified credible cases, which, subsequently, were investigated in primary care or referred for further investigation aiming for earlier diagnosis. This study also highlighted challenges in implementing such a tool, such as the restructuring of NHS organisations and shifting priorities due to outside pressures such as the COVID-19 pandemic.

Further research is ongoing, in the form of retrospective and prospective studies focusing on validating the algorithms and evaluating their analytical and clinical validity. Further studies to evaluate the cost-benefit of early diagnosis and the impact of MendelScan on clinical practice in primary care could prove beneficial from a payer perspective. A study is planned to give greater insight into the acceptability and perception of this novel approach among specialists, GP and patients.

A digital health approach, such as MendelScan, could be an invaluable tool to address the rare disease diagnostic odyssey, flagging those potential zebras amongst the horses. In addition, through the use of routinely collected EHR data, it can be scaled quickly and adopted broadly helping to ensure equality of access to a correct diagnosis.

\section{Declarations}

- Ethics approval and consent to participate: "Not applicable".

- Consent for publication: "Not applicable".

- Availability of data and materials: "Data sharing not applicable to this article as no datasets were generated or analysed during the current study".

- Funding: All funds were covered by Mendelian Ltd

- Competing interests: "The authors declare that they have no competing interests".

- Authors' contributions: OB created the algorithms and drafted the manuscript. SS drafted the manuscript. HM edited the manuscript. CT edited the manuscript. PR edited the manuscript, LM edited the manuscript, RB managed the data sharing agreement. WE participated in its design and coordination and helped to draft the manuscript. All authors read and approved the final manuscript. 
- Acknowledgements: "Not applicable"

\section{References}

1. Somanadhan S, Nicholson E, Dorris E, Brinkley A, Kennan A, Treacy E, Atif A, Ennis S, McGrath V, Mitchell D, O'Sullivan G, Power J, Lawlor A, Harkin P, Lynch SA, Watt P, Daly A, Donnelly S, Kroll T. Rare Disease Research Partnership (RAinDRoP): a collaborative approach to identify research priorities for rare diseases in Ireland. HRB Open Res. 2020 Nov 11;3:13. doi: 10.12688/hrbopenres.13017.2. PMID: 33299965; PMCID: PMC7702160.

2. Nguengang Wakap S, Lambert DM, Olry A, Rodwell C, Gueydan C, Lanneau V, Murphy D, Le Cam Y, Rath A. Estimating cumulative point prevalence of rare diseases: analysis of the Orphanet database. Eur J Hum Genet. 2020 Feb;28(2):165173. doi: 10.1038/s41431-019-0508-0. Epub 2019 Sep 16. PMID: 31527858; PMCID: PMC6974615.

3. Richter T, Nestler-Parr S, Babela R, Khan ZM, Tesoro T, Molsen E, Hughes DA; International Society for Pharmacoeconomics and Outcomes Research Rare Disease Special Interest Group. Rare Disease Terminology and Definitions-A Systematic Global Review: Report of the ISPOR Rare Disease Special Interest Group. Value Health. 2015 Sep;18(6):906-14. doi: 10.1016/j.jval.2015.05.008. Epub 2015 Aug 18. PMID: 26409619.

4. Moliner AM, Waligora J. The European Union Policy in the Field of Rare Diseases. Adv Exp Med Biol. 2017;1031:561-587. doi: 10.1007/978-3-319-67144-4_30. PMID: 29214592.

5. The UK Rare Diseases Framework [Internet]. GOV.UK. 2021 [cited 2021 May 18]. Available from: https://www.gov.uk/government/publications/uk-rare-diseases-framework/the-uk-rare-diseases-framework

6. [Internet]. Everylifefoundation.org. 2021 [cited 2021 May 26]. Available from: https://everylifefoundation.org/wpcontent/uploads/2021/02/The_National_Economic_Burden_of_Rare_Disease_Study_Summary_Report_February_2021.pdf

7. Kuiper, GA., Meijer, O.L.M., Langereis, E.J. et al. Failure to shorten the diagnostic delay in two ultra-orphan diseases (mucopolysaccharidosis types I and III): potential causes and implications. Orphanet J Rare Dis 13, 2 (2018). https://doi.org/10.1186/s13023-017-0733-y

8. Zurynski, Y., Deverell, M., Dalkeith, T. et al. Australian children living with rare diseases: experiences of diagnosis and perceived consequences of diagnostic delays. Orphanet J Rare Dis 12, 68 (2017). https://doi.org/10.1186/s13023-0170622-4

9. A cohort of patients with RD in the UK and US reported a diagnostic delay of an average 5.6 and 7.6 years respectively, with patients typically visiting eight physicians (four primary care and four specialists) and receiving two to three misdiagnoses

10. [Internet]. Eurordis.org. 2021 [cited 2021 May 25]. Available from: https://www.eurordis.org/IMG/pdf/Fact_Sheet_Eurordiscare2.pdf

11. Sotos John. Dr. Zebra. The Celebrated Web Site. The clinical medicine classic Zebra Cards. http://www.sotos.com/ (accessed 21 May 2021). [Google Scholar]. [Ref list]

12. Evans WR, Rafi I. Rare diseases in general practice: recognising the zebras among the horses. Br J Gen Pract. 2016 Nov;66(652):550-551. doi: 10.3399/bjgp16X687625. PMID: 27789486; PMCID: PMC5072891.

13. Laraway S, Breen C, Mercer J, Jones S, Wraith JE. Does early use of enzyme replacement therapy alter the natural history of mucopolysaccharidosis I? Experience in three siblings. Mol Genet Metab. 2013 Jul;109(3):315-6. doi:

10.1016/j.ymgme.2013.04.023. Epub 2013 May 10. PMID: 23721889.

14. Kohlschütter $A$, van den Bussche H. Frühzeitige Diagnose einer seltenen Krankheit bei Kindern durch bessere Kommunikation zwischen Eltern, niedergelassenen Ärzten und spezialisierten Zentren [Early diagnosis of a rare disease in children through better communication between parents, physicians and academic centers]. Z Evid Fortbild Qual Gesundhwes. 2019 May;141-142:18-23. German. doi: 10.1016/j.zefq.2019.02.008. Epub 2019 Mar 14. PMID: 30878243.

15. Ng DM, Hooper AJ, Bellgard MI, Burnett JR. The role of patient registries for rare genetic lipid disorders. Curr Opin Lipidol. 2018 Apr;29(2):156-162. doi: 10.1097/MOL.0000000000000485. PMID: 29351106.

16. Assets.publishing.service.gov.uk. 2021 [cited 2021 May 26]. Available from: https://assets.publishing.service.gov.uk/government/uploads/system/uploads/attachment_data/file/950651/the-UK-rare- 
diseases-framework.pdf

17. SNOMED CT - NHS Digital [Internet]. NHS Digital. 2021 [cited 2021 Jun 3]. Available from: https://digital.nhs.uk/services/terminology-and-classifications/snomed-ct

18. Mendelian.co. 2021 [cited 2021 Jun 17]. Available from: https://www.mendelian.co/pdf/ethics-report-ph-mendelian.pdf

19. Data protection impact assessments [Internet]. Ico.org.uk. 2021 [cited 2021 Jun 3]. Available from: https://ico.org.uk/fororganisations/guide-to-data-protection/guide-to-the-general-data-protection-regulation-gdpr/accountability-andgovernance/data-protection-impact-assessments/

20. Zahabi M , Kaber DB , Swangnetr M . Usability and safety in electronic medical records interface design: a review of recent literature and guideline formulation. Hum Factors 2015;57:805-34.doi:10.1177/0018720815576827

21. Hayward J , Thomson F, Milne H, et al . 'Too much, too late': mixed methods multi-channel video recording study of computerized decision support systems and GP prescribing. J Am Med Inform Assoc 2013;20:e76e84.doi:10.1136/amiajnl-2012-001484

22. Backman R, Bayliss S, Moore D, Litchfield I. Clinical reminder alert fatigue in healthcare: a systematic literature review protocol using qualitative evidence. Syst Rev. 2017 Dec 13;6(1):255. doi: 10.1186/s13643-017-0627-z. PMID: 29237488; PMCID: PMC5729261.

\section{Tables}

Table 1 - Electronic health record (EHR) code types used.

\begin{tabular}{|ll|}
\hline EHR code type & Description \\
\hline Vitals & Vital values such as blood pressure, weight, height, ethnicity and BMI \\
\hline Demographics & Patients demographic information such as age and sex \\
\hline Problem list & Patients list of active medical issues \\
\hline Past medical history & Diagnosis of chronic conditions diagnosed previously \\
\hline Diagnosis & Diagnosis and diagnostic codes \\
\hline Referrals & Referral ordered and admissions \\
\hline Medications & Medications ordered and currently taken \\
\hline Interventions & Interventions requested and intervention results \\
\hline Lab results & Numerical results of any laboratory tests \\
\hline
\end{tabular}

Table 4 - First feedback outcomes

\begin{tabular}{|lllll|}
\hline & $\begin{array}{l}\text { Reasonable possible } \\
\text { diagnosis (advanced for } \\
\text { investigation) }\end{array}$ & $\begin{array}{l}\text { Diagnosis has } \\
\text { already been } \\
\text { excluded }\end{array}$ & $\begin{array}{l}\text { GP believes the patient } \\
\text { has a clear alternative } \\
\text { aetiology }\end{array}$ & $\begin{array}{l}\text { Other (Patient no longer at the } \\
\text { practice or unable to correctly } \\
\text { identify patient record }\end{array}$ \\
\hline $\begin{array}{l}\text { \# of } \\
\text { patients }\end{array}$ & 9 & 6 & 10 & 3 \\
\hline
\end{tabular}

Table 5 -Second feedback outcomes 


\begin{tabular}{|llll|}
\hline $\begin{array}{l}\text { Patient } \\
\text { EHR }\end{array}$ & Suggested disease & Action taken by GP & Outcome \\
\hline 1 & Classic Homocystinuria & Discuss with patient & Pending \\
\hline 2 & Fabry DIsease & Discuss with patient & Pending \\
\hline 3 & Alpha-1 antitrypsin deficiency & $\begin{array}{l}\text { Alpha-1-antitrypsin } \\
\text { level }\end{array}$ & Normal \\
\hline 4 & Alpha-1 antitrypsin deficiency & $\begin{array}{l}\text { Alpha-1-antitrypsin } \\
\text { level }\end{array}$ & Normal \\
\hline 5 & Loeys-Dietz syndrome & Referral to cardiologist & $\begin{array}{l}\text { Seen by Cardiologist, pending } \\
\text { cardiologist letter }\end{array}$ \\
\hline 6 & Loeys-Dietz syndrome & Referral to cardiologist & Seen by Cardiologist, pending \\
& Beckwith-Wiedemann syndrome & Discuss with patient & Pending \\
\hline 7 & Alpha-1 antitrypsin deficiency & $\begin{array}{l}\text { Alpha-1-antitrypsin } \\
\text { level }\end{array}$ & Normal \\
\hline 9 & $\begin{array}{l}\text { DiGeorge Syndrome - 22q11 deletion } \\
\text { syndrome }\end{array}$ & $\begin{array}{l}\text { Referral to clinical } \\
\text { geneticist }\end{array}$ & 1. Seen by Clinical geneticist \\
\hline
\end{tabular}

\section{Supplementary Files}

This is a list of supplementary files associated with this preprint. Click to download.

- 7Appendix.docx 